\title{
Small RNAs derived from snoRNAs
}

\author{
RYAN J. TAFT, ${ }^{1}$ EVGENY A. GLAZOV, ${ }^{2}$ TIMO LASSMANN, ${ }^{3,4}$ YOSHIHIDE HAYASHIZAKI, $^{3,4}$ \\ PIERO CARNINCI, ${ }^{3,4}$ and JOHN S. MATTICK ${ }^{1}$ \\ ${ }^{1}$ Australian Research Council Special Research Centre for Functional and Applied Genomics, Institute for Molecular Bioscience, The University \\ of Queensland, St. Lucia, QLD 4072, Australia \\ ${ }^{2}$ Diamantina Institute for Cancer, Immunology and Metabolic Medicine, The University of Queensland, Princess Alexandra Hospital, \\ Woolloongabba, QLD 4102, Australia \\ ${ }^{3}$ Genome Exploration Research Group, RIKEN Omics Science Center and the Functional RNA Research Program, Frontier Research System, \\ RIKEN Yokohama Institute, Yokohama, Kanagawa, 230-0045 Japan \\ ${ }^{4}$ Genome Science Laboratory, Discovery and Research Institute, RIKEN Wako Institute, 2-1 Hirosawa, Wako-shi, Saitama, $351-0198$ Japan
}

\begin{abstract}
Small nucleolar RNAs (snoRNAs) guide RNA modification and are localized in nucleoli and Cajal bodies in eukaryotic cells. Components of the RNA silencing pathway associate with these structures, and two recent reports have revealed that a human and a protozoan snoRNA can be processed into miRNA-like RNAs. Here we show that small RNAs with evolutionary conservation of size and position are derived from the vast majority of snoRNA loci in animals (human, mouse, chicken, fruit fly), Arabidopsis, and fission yeast. In animals, sno-derived RNAs (sdRNAs) from H/ACA snoRNAs are predominantly 20-24 nucleotides (nt) in length and originate from the $3^{\prime}$ end. Those derived from C/D snoRNAs show a bimodal size distribution at $\sim$ 17-19 $\mathrm{nt}$ and $>27 \mathrm{nt}$ and predominantly originate from the $5^{\prime}$ end. SdRNAs are associated with AGO7 in Arabidopsis and Ago1 in fission yeast with characteristic $5^{\prime}$ nucleotide biases and show altered expression patterns in fly loquacious and Dicer-2 and mouse Dicer1 and Dgcr8 mutants. These findings indicate that there is interplay between the RNA silencing and snoRNAmediated RNA processing systems, and that sdRNAs comprise a novel and ancient class of small RNAs in eukaryotes.
\end{abstract}

Keywords: small nucleolar RNA; microRNA; deep sequencing; Argonaute; Dicer

\section{INTRODUCTION}

Small nucleolar RNAs are a highly evolutionarily conserved class of RNAs, which are present throughout the Eukaryotes and whose origins lie in the Archaea (Gaspin et al. 2000; Omer et al. 2000; Matera et al. 2007). There are two classes of snoRNAs (C/D and H/ACA box) that function as ribonucleoprotein (RNP) complexes to guide the enzymatic modification of target RNAs at sites determined by RNA:RNA antisense interactions (Matera et al. 2007). Generally, C/D box snoRNAs are $\sim 70-120$ nucleotides (nt) and guide the methylation of target RNAs, while $\mathrm{H}$ / ACA box snoRNAs are $\sim 100-200$ nt and guide pseudouridulation (Matera et al. 2007). These RNAs were initially discovered in the nucleolus and thought to exclusively target ribosomal RNAs, but are now recognized to be a

Reprint requests to: John S. Mattick, Australian Research Council Special Research Centre for Functional and Applied Genomics, Institute for Molecular Bioscience, The University of Queensland, St. Lucia, QLD 4072, Australia; e-mail: j.mattick@imb.uq.edu.au; fax: 61733462111.

Article published online ahead of print. Article and publication date are at http://www.rnajournal.org/cgi/doi/10.1261/rna.1528909. much broader class of RNAs with different functions, targets, and subcellular locations (Matera et al. 2007).

Argonaute proteins are key players in the RNA silencing pathways, including the microRNA (miRNA), small interfering RNA (siRNA), and PIWI-associated RNA-(piRNA) mediated regulation of gene expression (Peters and Meister 2007; Hutvagner and Simard 2008). Recent proteomic and immunofluorescence analyses of Argonaute-containing RNA-protein complexes in animals have indicated that there may be a link between the RNA silencing and snoRNA pathways (Hock et al. 2007; Hutvagner and Simard 2008). Mass spectrometry of human HEK-293 cell Argonaute-associated proteins revealed a direct interaction between AGO1 and AGO2, and Nop56 and Fibrillarin, respectively, both of which are components of the snoRNA RNP complex (Kiss 2002; Hock et al. 2007; Matera et al. 2007). In plants, at least four key components of the RNAinduced silencing complex (RNA DEPENDENT RNA POLYMERASE 2, DICER-LIKE 3, ARGONAUTE4, and the largest subunit of Pol IVb, NRPD1b) localize to the nucleolus (Li et al. 2006, 2008; Pontes et al. 2006), the primary site of snoRNA biogenesis and function, and concentrate in 
Cajal bodies to assemble Ago-siRNA complexes (Pontes et al. 2006; Pontes and Pikaard 2008). Moreover, recent reports have indicated that a human snoRNA and a protozoan snoRNA are associated with Argonaute, processed into small RNAs, and can function as miRNAs (Ender et al. 2008; Saraiya and Wang 2008).

\section{RESULTS AND DISCUSSION}

We performed a systematic analysis of small RNA libraries we generated from a human monocytic leukemia cell line (THP-1) (Taft et al. 2009) and embryonic chicken (Glazov et al. 2008) and found extensive evidence for small RNAs derived from both classes of snoRNA. We then extended our analyses to other small-RNA deep sequencing libraries, including those from mouse embryonic stem (ES) cells (Babiarz et al. 2008), a variety of Drosophila tissues and life-cycle stages (Ruby et al. 2007; Czech et al. 2008), Arabidopsis ARGONAUTE2 and ARGONAUTE7 coimmunoprecipitations (IPs) (Montgomery et al. 2008), and Argo-

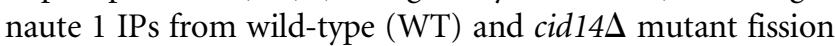
yeast Schizosaccharomyces pombe (Supplemental Tables 1, 2; Buhler et al. 2008). More than $60 \%$ of human and mouse,
80\% of chicken and Drosophila, and nearly all Arabidopsis and fission yeast snoRNAs show evidence of highly conserved sdRNAs (Supplemental Fig. 1), which occupy $\sim 0.1$ to $>5 \%$ of total deep sequencing reads (Supplemental Table 1). In human THP-1 cells, we found that many highly abundant sdRNAs are derived from weakly expressed snoRNAs, suggesting that sdRNAs are the result of regulated processing rather than RNA turnover (Supplemental Fig. 2).

Indeed, metazoan sdRNAs exhibit other characteristics suggestive of regulated biogenesis, including distinct size distributions. Those derived from C/D box snoRNAs (C/D sdRNAs) are predominantly $\sim 17-19 \mathrm{nt}$ and $\sim 30 \mathrm{nt}$ (Fig. 1 ), the latter of which is typical of piRNAs (Peters and Meister 2007). These piRNA-sized C/D sdRNAs are detectable by Northern (Supplemental Fig. 3), but are only directly observable in the human data set, which contains deep sequencing reads extending up to $90 \mathrm{nt}$ (see Materials and Methods). H/ACA box snoRNA-derived RNAs (H/ ACA sdRNAs) show a peak at $\sim 22 \mathrm{nt}$, similar to miRNAs, although in mouse this may be affected by contextdependent regulation of sdRNA biogensis (see below) (Fig. 1 ), and in flies, there are significant numbers of 23-24-nt small RNAs that may be related to small RNAs associated
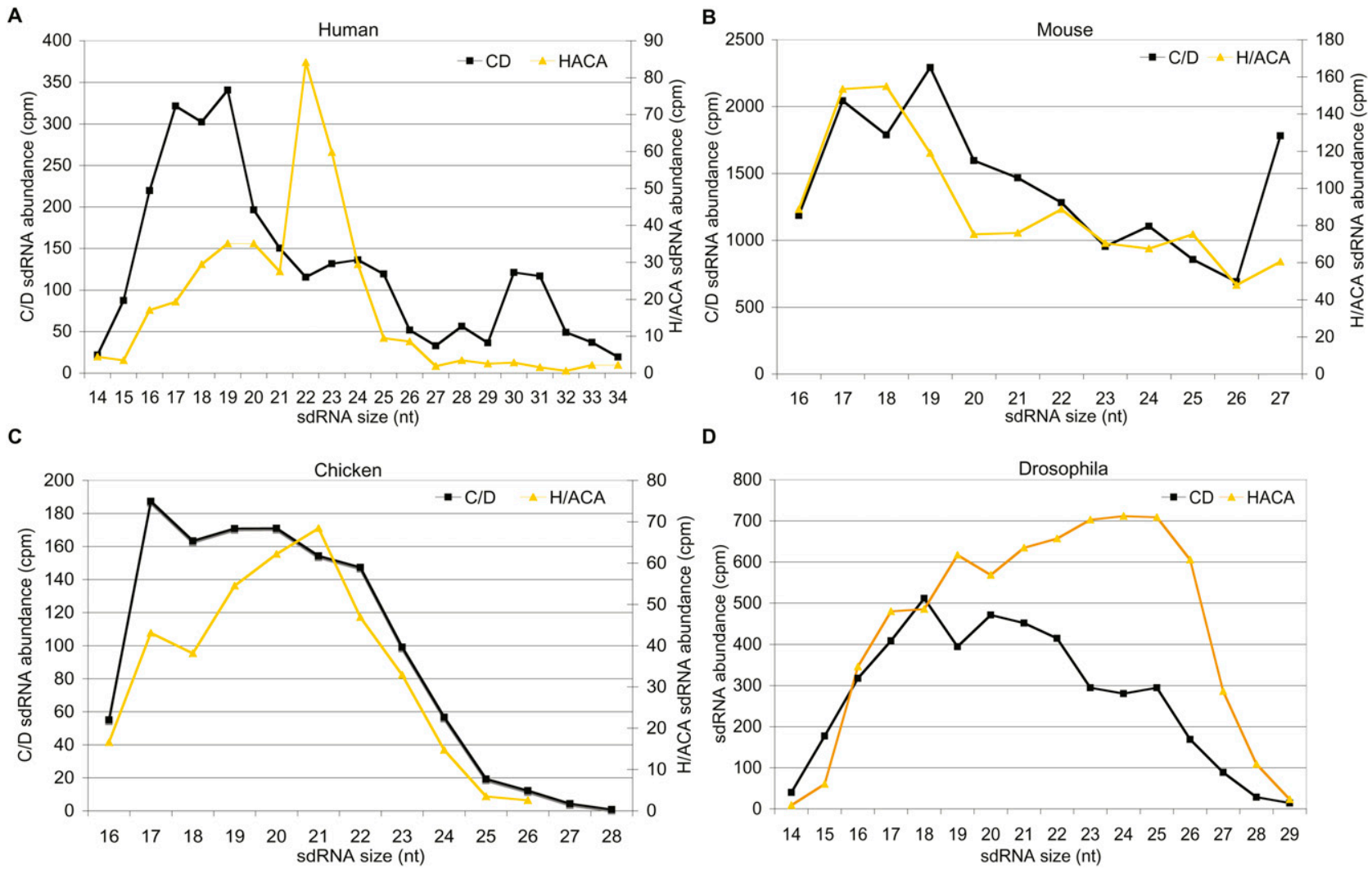

FIGURE 1. sdRNAs size distributions in metazoa. (Black) C/D and (yellow) H/ACA sdRNA sizes are plotted for $(A)$ human, $(B)$ mouse, $(C)$ chicken, and (D) Drosophila. In all species, sdRNAs segregate by size dependent on the snoRNA type they originate from. In animals, C/D sdRNAs are more highly expressed and smaller than H/ACA sdRNAs. Vertical axes are in units of counts per million (cpm) - a ratio of the abundance of sdRNAs per million mapped tags in the total library. In $A-C, \mathrm{C} / \mathrm{D}$ sdRNA abundance is denoted on the left of each panel, $\mathrm{H} / \mathrm{ACA}$ on the right. 
with AGO4 and Cajal bodies, one of the sites of H/ACA snoRNA function (Fig. 1; Li et al. 2008).

Further evidence of the specificity of sdRNAs is provided by the observation that $\mathrm{C} / \mathrm{D}$ sdRNAs are derived predominantly from the $5^{\prime}$ end of full-length snoRNAs, whereas H/ACA sdRNAs are derived predominantly from the $3^{\prime}$ end (Fig. 2), both of which form double-stranded RNA structures (Matera et al. 2007). These data indicate a strong tendency for sdRNAs to be derived from the $3^{\prime}$ arm of one of the two miRNA-like hairpins that compose $\mathrm{H}$ / ACA snoRNAs (Fig. 2E). We also observed processing specificity for $\sim 30$-nt human C/D sdRNAs that originate from the middle of the host snoRNA (Supplemental Fig. 4). Sno-derived RNA size and position distributions are conserved in human, mouse, chicken, and Drosophila (Supplemental Fig. 5). Indeed, orthologous sdRNAs are identifiable in all three vertebrate species at 31 snoRNA loci (26 C/D and $5 \mathrm{H} / \mathrm{ACA}$ ) and in two or more vertebrate species at 141 loci (103 C/D and $38 \mathrm{H} / \mathrm{ACA})$, and exhibit conservation of size and position within the host snoRNA, albeit with some terminal sequence heterogeneity, which is typical of other types of small RNAs, including miRNAs (Supplemental Fig. 5; Wu et al. 2007; Morin et al. 2008).

To determine if RNAi pathway proteins affect the biogenesis of sdRNAs, we examined small RNA libraries from dicer $1 \Delta / \Delta$ and $\operatorname{dgcr} 8 \Delta / \Delta$ mouse ES cells (Babiarz et al. 2008), and loquacious (loqs) and Dicer-2 (Dcr-2) mutant flies (Czech et al. 2008). Dicer1 and DGCR8 are principal
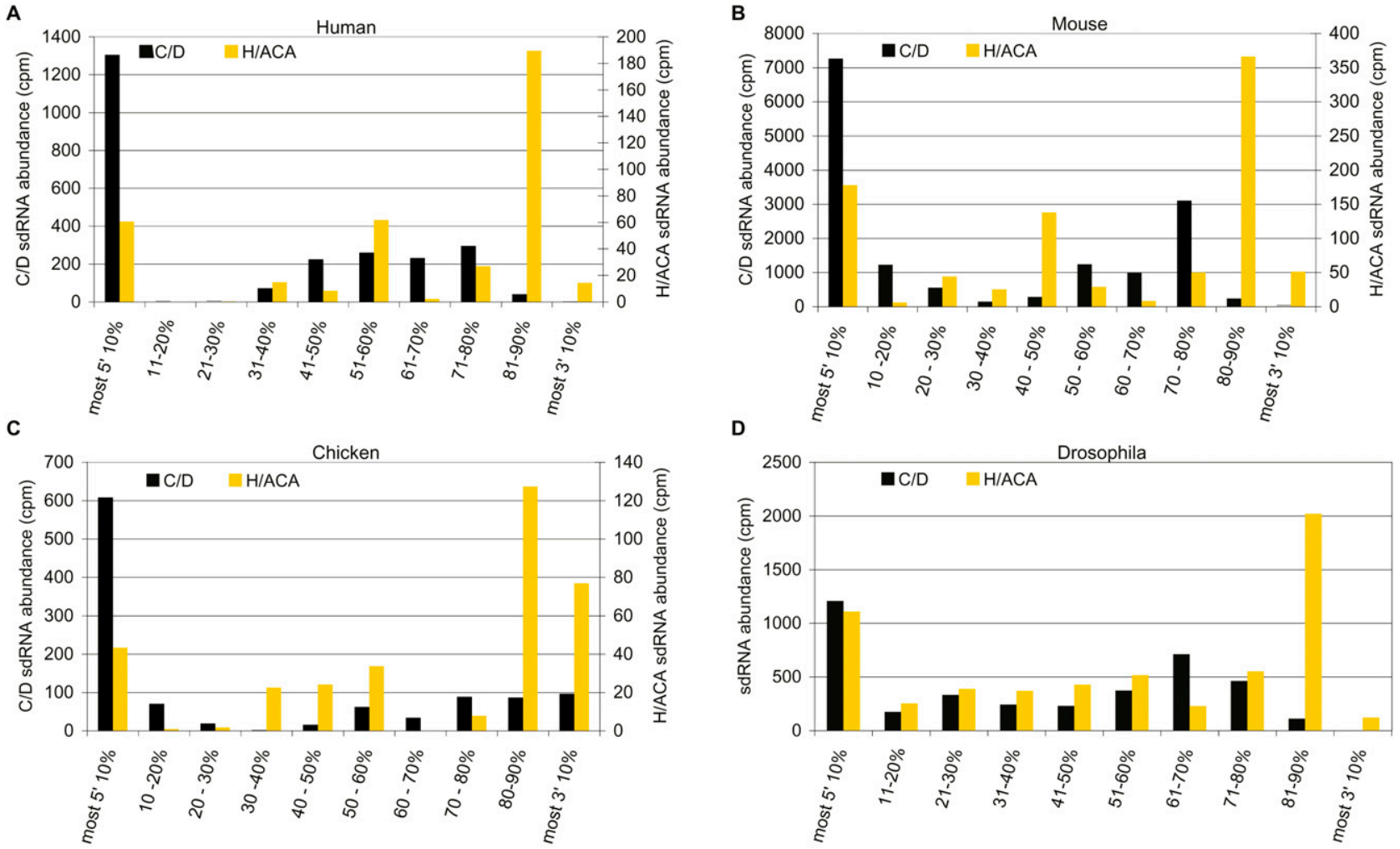

E Mouse ACA58b

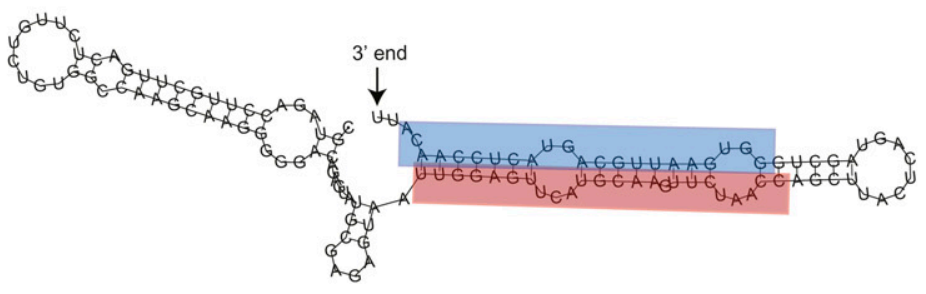

FIGURE 2. sdRNA position-of-origin within parent snoRNAs. The position-of-origin of (black) C/D and (yellow) H/ACA is shown for ( $A$ ) human, $(B)$ mouse, $(C)$ chicken, and $(D)$ Drosophila. To normalize for varying host snoRNA lengths, we divided snoRNAs into $10 \%$ blocks (deciles) from the $5^{\prime}$ to the $3^{\prime}$ end, and plotted the positions of the $5^{\prime}$ end of each sdRNA. C/D sdRNAs are primarily derived from the $5^{\prime}$ end of snoRNAs. H/ACA sdRNAs are predominantly derived from the $3^{\prime}$ end, a position that correlates to one of the two H/ACA snoRNA hairpins. C/D sdRNA abundance is denoted on the left of each panel, H/ACA on the right. (E) The structure of mouse ACA58b. SdRNAs are predominantly localized to the $3^{\prime}$ end of the RNA secondary structure. Sequences shaded in red and blue indicate miRNA-like mature and star sequences, respectively. 
components of the miRNA biogenesis pathway in mammals. Pre-miRNA hairpins are cleaved from primary miRNA transcripts by Drosha-DGCR8 complexes and then processed into $\sim 22$-nt miRNAs by Dicer1. Consistent with the original report describing these data (Babiarz et al. 2008), we normalized against the abundance of tRNA, snRNA, and srpRNAs in each library (see Materials and Methods; Supplemental Table 3 ), which implicitly corrects for varying library depth, the loss of specific small RNA (e.g., miRNA or siRNA) species, and varying amounts of RNA degradation. The proportions of miRNAs in these libraries are $\sim 100$-fold and 20 fold less abundant in dicer $1 \Delta / \Delta$ and $d g c r 8 \Delta / \Delta$ ES cells, respectively. In contrast, C/D sdRNAs are only mildly down-regulated and show size distributions identical to wild type (Fig. 3A).

H/ACA sdRNAs, however, show pronounced responses to the loss of Dicer1 and $D g c r 8$. SnoRNA-derived small RNAs from two H/ACA loci are more than fourfold down-regulated in the absence of Dicerl, and a further nine are at least twofold down-regulated. Intriguingly, three, including those that are the most affected by the loss of Dicer, are twofold up-regulated in the absence of $D g c r 8$ (Fig. 3B), suggesting that in some cases, DGCR8 may serve as an inhibitor of small RNA biogenesis. Indeed, $d g c r 8 \Delta / \Delta$ H/ACA sdRNAs exhibit a dominant $\sim 22$-nt peak, similar to known miRNAs, with $>2.5$-fold increases in the number of 22 and 23 mers compared with wild type, which is primarily driven by three snoRNAs (Fig. 3B, ACA36b, ACA58b, ACA41). These data indicate that sdRNAs derived from at least two H/ACA snoRNAs are Dicer1 sensitive and that DGCR8 may negatively regulate a subset of H/ACA sdRNAs. Indeed, H/ACA snoRNAs are principally composed of two short miRNA-like hairpins, which would appear to be ideal DrohaDGCR8 or Dicer substrates. Consistent with this hypothesis, the snoRNA ACA36b was recently annotated as a Dicersensitive endogenous shRNA (Babiarz et al. 2008) capable of generating miRNA-like small RNAs (Ender et al. 2008).

Endogenous 21-nt siRNAs have been described in Drosophila and mammals (Babiarz et al. 2008; Czech et al. 2008; Ghildiyal et al. 2008; Watanabe et al. 2008). In flies, siRNAs are Dcr-2 dependent, although a subset also relies on

B one library.
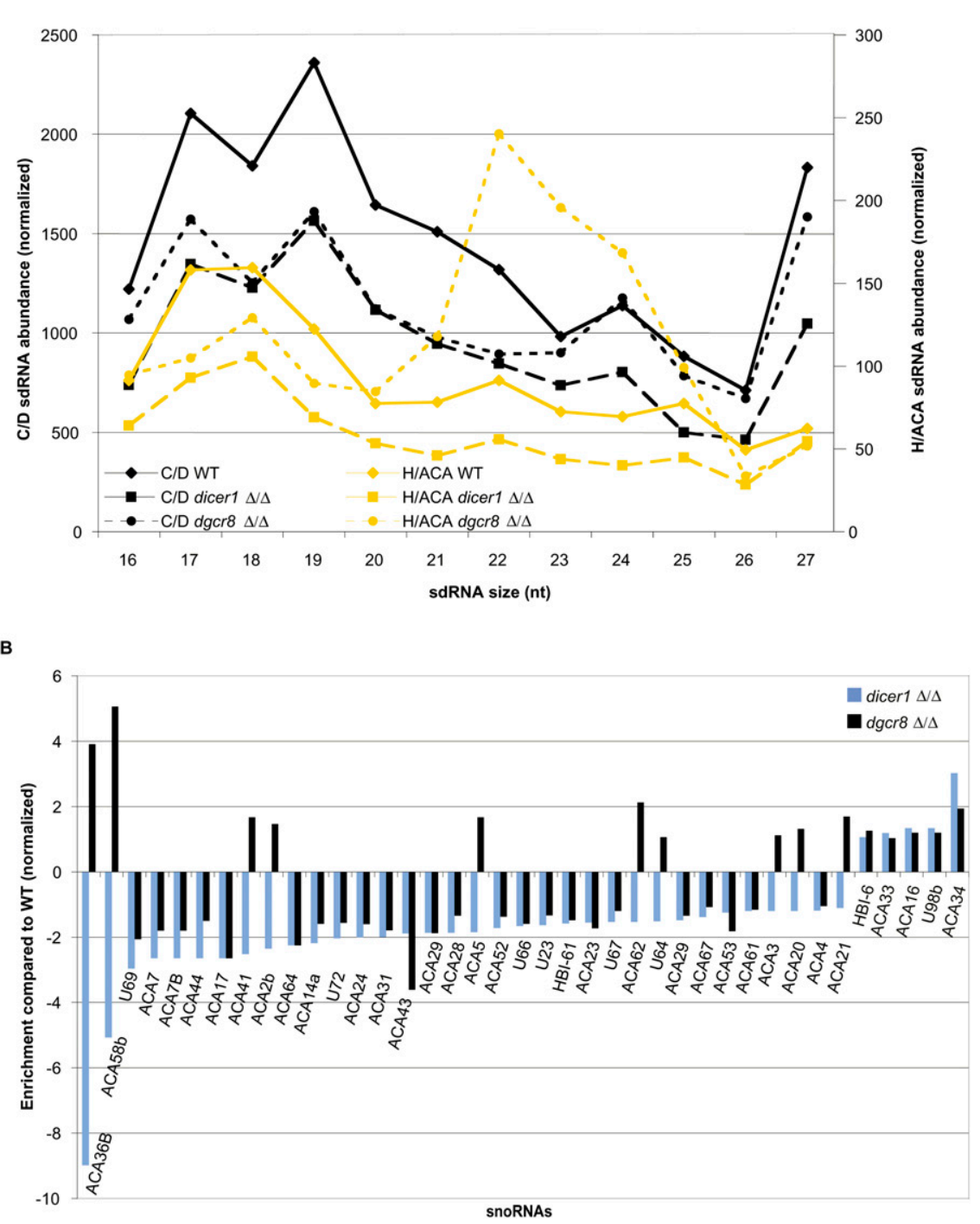

FIGURE 3. Mouse sdRNAs are affected by the loss of Dicer1 and Dgcr8. Wild-type and mutant ES cell libraries were normalized to t/sn/srpRNA abundance (Supplemental Table 4; see the text). (A, black) $\mathrm{C} / \mathrm{D}$ and (yellow) H/ACA sdRNA sizes in (solid lines) wild-type (WT), (large dashed lines) dicer $1 \Delta / \Delta$, and (small dashed lines) $d g c r 8 \Delta / \Delta$ embryonic stem cells. H/ACA sdRNAs also exhibit an increase of 21-24-nt species in $d g c r 8 \Delta / \Delta$ ES cells. (B) The relative enrichment of sdRNA abundance compared to WT of $39 \mathrm{H} / \mathrm{ACA}$ snoRNAs in (blue) dicer $1 \Delta / \Delta$ and (black) $d g c r 8 \Delta / \Delta$ embryonic stem cells. SnoRNAs were only included in the analysis if their sdRNAs had an abundance greater than 5 in each library and greater than 10 in at least

the double-stranded RNA-binding protein and Dicer partner, loquacious. We normalized small RNA libraries from mutant flies to $\mathrm{t} / \mathrm{sn} / \mathrm{srpRNA}$ fragments (Supplemental Table 4; see Materials and Methods) and found that $\mathrm{C} / \mathrm{D}$ and $\mathrm{H} /$ ACA sdRNAs are more than fivefold, up-regulated in $l o q s^{-1-}$ ovaries (Supplemental Figs. 6, 7) and show characteristics consistent with other Drosophila libraries (Supplemental Fig. 6), suggesting that the absence of loquacious leads to specific sdRNA enrichment. Indeed, sdRNAs from some snoRNA loci are more than 35-fold up-regulated (Supplemental Fig. 7). Additionally, sdRNAs from nine $\mathrm{C} / \mathrm{D}$ and three H/ACA 
snoRNAs are more than twofold down-regulated in the absence of Dicer-2 (Supplemental Fig. 7). These data provide further evidence that sdRNA biogenesis is regulated by components of the RNAi pathway including proteins conventionally associated with siRNAs.

MiRNAs and siRNAs associate with the Argonaute family of proteins (Peters and Meister 2007; Hutvagner and Simard 2008). To test if sdRNAs are Argonaute associated, we queried AGO2 and AGO7 libraries from Arabidopsis (Montgomery et al. 2008) and Agol libraries from S. pombe (Buhler et al. 2008). We found that sdRNAs in Arabidopsis are strongly associated with AGO7 but not AGO2 (Fig. 4A; Supplemental Fig. 8). Eight H/ACA box and $25 \mathrm{C} / \mathrm{D}$ box snoRNAs are more than twofold enriched in the AGO7 library, corresponding to $\sim 4 \%$ of all AGO7 associated small RNAs (Fig. 4A). Like metazoan sdRNAs, AGO7 H/ACA and C/D sdRNAs differ in size. C/D sdRNAs are smaller and dominantly $21 \mathrm{nt}$, while H/ACA sdRNAs are larger, 27-29 nt (Supplemental Fig. 9). Arabidopsis Argonaute proteins preferentially load small RNAs with specific $5^{\prime}$ nucleotides and

A

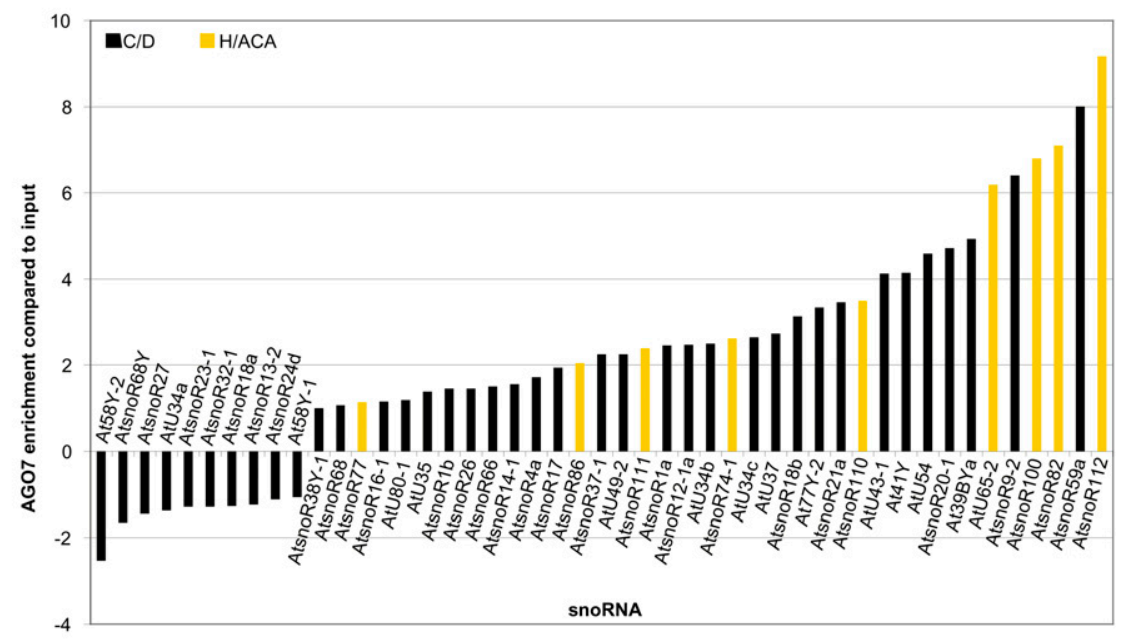

B
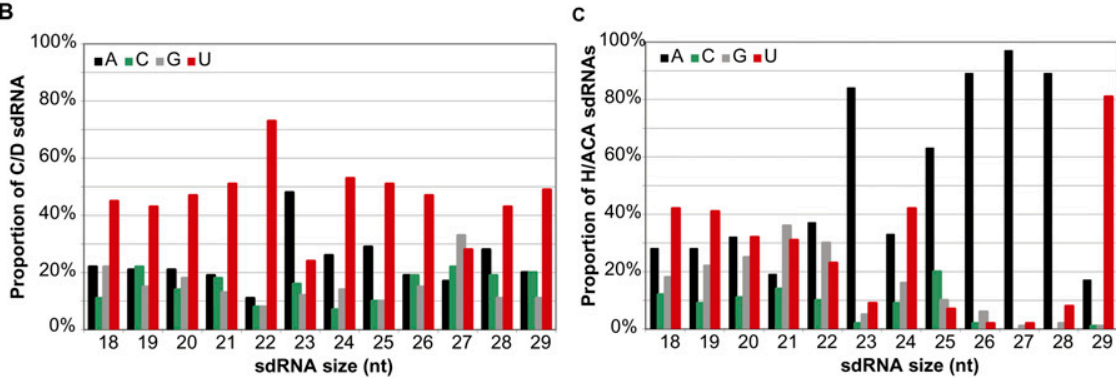

FIGURE 4. sdRNAs are associated with Arabidopsis AGO7. (A) The relative enrichment of (black) C/D and (yellow) H/ACA sdRNA abundance from individual snoRNAs in Arabidopsis AGO7 immunoprecipitations. Enrichments are calculated in comparison to IP input deep sequencing libraries. SnoRNAs were only included in the analysis if their sdRNAs had an abundance greater than 5 in each library and greater than 10 in at least one library. H/ACA and C/D sdRNAs are strongly enriched in the AGO7 library. $(B, C)$ The proportions of C/D and H/ ACA sdRNAs $5^{\prime}$ end nucleotides associated with Arabidopsis AGO7. C/D sdRNAs are $5^{\prime} \mathrm{U}$ biased and H/ACA sdRNAs are 5'A biased.
AGO7 is selective for $5^{\prime}$ uracil (U) and $5^{\prime}$ adenine (A). Intriguingly, $\mathrm{H} / \mathrm{ACA}$ sdRNAs are dominantly $5^{\prime} \mathrm{A}$, while C/D box are dominantly $5^{\prime} \mathrm{U}$ (Fig. $4 \mathrm{~A}, \mathrm{~B}$ ).

We observed similar trends in fission yeast. S. pombe Ago1-associated snoRNA fragments were recently reported in $\mathrm{WT}$ and cid14s [a non-canonical poly(A) polymerase and member of the exosome-mediated RNA degradation pathway] cells (Buhler et al. 2008). Consistent with previous findings, we find that sdRNAs comprise $\sim 1.6 \%$ of the WT library and are up-regulated almost twofold to $\sim 3.3 \%$ of the cid14s library (Supplemental Fig. 10; Supplemental Table 1). H/ACA sdRNAs dominate both Agol libraries (greater than fourfold over C/D sdRNAs), and show an up-regulation of 20-nt small RNAs in cid14D cells (Supplemental Fig. 11) that are derived from the central portion of the host snoRNA and fall on the $5^{\prime}$ arm of the $3^{\prime}$ hairpin (Supplemental Fig. 11). C/D sdRNA sizes are dominantly 22 and $23 \mathrm{nt}$ in both data sets (similar to the library as a whole) (Supplemental Fig. 11), and unlike metazoan C/D sdRNAs, are principally derived from the $3^{\prime}$ end of the parent snoRNA (Supplemental Fig. 12). Like Argonautes in higher eukaryotes, S. pombe Agol preferentially loads small RNAs with a $5^{\prime} \mathrm{U}$ (Montgomery et al. 2008). Congruent with the $5^{\prime}$ nucleotide bias observed in Arabidopsis, we found that the vast majority of C/D and H/ACA sdRNAs of all sizes are $5^{\prime} \mathrm{U}$ enriched (Supplemental Fig. 13). These data strongly suggest that Arabidopsis and fission yeast sdRNAs are preferentially loaded into Argonaute complexes, which has also recently been reported for a small set of human sdRNAs (Ender et al. 2008), and are likely to have roles in the regulation of gene expression and transcriptional silencing. In particular, the fact that both miRNAs and sdRNAs are $5^{\prime} U$ biased strengthens the link between them and suggests that some snoRNAs, including "orphan" snoRNAs whose targets are unknown, may function solely as intermediates in the sdRNA pathway.

The findings presented here indicate that sno-derived small RNAs are a general feature of both animal and plant biology, and that snoRNAs may have an ancient link with RNA silencing (Saraiya and Wang 2008), given that (1) sdRNAs are found in vertebrates, invertebrates, plants, and unicellular eukaryotes; (2) show a characteristic size distribution and origin that is distinctive with respect 
to the two major types of snoRNAs; (3) show specific responses to the loss of components of the RNAi pathway; and (4) are associated with specific members of the Argonaute family of proteins. It also reinforces the likelihood of an interplay between the RNA silencing and snoRNAmediated RNA processing and RNA-directed regulatory systems. Indeed, given that snoRNAs are active in the nucleolus, the strong association of $\mathrm{C} / \mathrm{D}$ and H/ACA sdRNAs with Argonautes is consistent with the emerging picture of the nucleolus as a site of small RNA biogenesis, stabilization, and function in animals, plants, and fungi (Pontes and Pikaard 2008). Additionally, sdRNA upregulation in the absence of DGCR8, loquacious, and Cid14 suggests that sdRNA biogenesis is tightly controlled by known dsRNA binding proteins and members of the exosome-mediated small RNA processing pathway. These findings also suggest that approaches combining comparative genomics and deep sequencing are capable of revealing new classes of small RNAs and that snoRNAs, like other small RNAs, may be more multifaceted than previously supposed.

\section{MATERIALS AND METHODS}

\section{Additional small RNA data sets}

Small RNAs were obtained from 26 publicly available small RNA deep sequencing libraries (identifiers are listed in parentheses): human THP-1 small RNAs (DNA Database of Japan, AIAAA0000001-AIAAT0000001) (Taft et al. 2009); mouse WT, dicer $1 \Delta / \Delta$, and $d g c r 8 \Delta / \Delta$ ES stem cells (NCBI GEO, GSE12521) (Babiarz et al. 2008); chicken libraries from embryonic days 5, 7, and 9 (NCBI GEO, GSE10686) (Glazov et al. 2008); a variety of Drosophila tissues and life-cycle stages, and $\mathrm{Dcr}-2^{-1-}$ and $l o q s^{-1-}$ mutants (NCBI GEO, GSE7448 and GSE11086) (Ruby et al. 2007; Czech et al. 2008); Arabidopsis ARGONAUTE4 and ARGONAUTE7 IPs (NCBI GEO, GSE12037) (Montgomery et al. 2008); and Argonaute-1 IPs from WT and cid14D mutant $S$. pombe (NCBI GEO, GSE311595) (Buhler et al. 2008).

\section{Small nucleolar RNA annotations}

SnoRNA annotations were compiled from multiple sources. Human snoRNA annotations were obtained through the small RNA UCSC Genome Browser track (wgRNA) (Karolchik et al. 2008). Mouse snoRNAs were compiled from two sources: (1) we obtained a set of curated snoRNAs from the NCBI Entrez Gene database ( $\sim 50$ snoRNAs) (Wheeler et al. 2008); and (2) we identified orthologous evolutionary conserved mouse snoRNAs by mapping the human snoRNA annotations to the mouse genome using the UCSC syntenic alignment program, liftOver, requiring 95\% sequence identity. Chicken snoRNAs annotations were obtained from the Ensemble chicken genome database (Flicek et al. 2008). The complete set of Drosophila snoRNAs was obtained from FlyBase (Drysdale 2008). Arabidopsis snoRNA annotations from Brown et al. (2001) were obtained from the Lowe Lab snoRNA database website (http://lowelab.ucsc.edu/snoRNAdb/). $S$. pombe snoRNA annotations were obtained from the Sanger Institute's GeneDB (Hertz-Fowler et al. 2004). Human, mouse, chicken, and Drosophila snoRNAs C/D and H/ACA box annotations were derived from the data files described above. Arabidopsis and $S$. pombe snoRNAs were manually curated for $\mathrm{C} / \mathrm{D}$ and $\mathrm{H} /$ ACA annotations by querying the Entrez Gene and NCBI nucleotide databases with the sequence of interest using BLAST. A subset of $S$. pombe snoRNAs without snoRNA-type annotations were designated as C/D or H/ACA based on the presence of canonical sequence motifs and total sequence length, yielding a total of 17 and 27 high-confidence C/D and H/ACA box S. pombe snoRNAs, respectively.

\section{sdRNA analysis}

Bioinformatics analysis was done on a high-performance computing station that houses a local mirror of the UCSC Genome Browser (Karolchik et al. 2008) and a local installation of the Biopieces toolset (http://www.biopieces.org/, developed by Martin Hansen). All small RNA data sets were mapped onto reference genomes using Vmatch (http://www.vmatch.de/). We used the UCSC builds of the following genome assemblies: human (hg18 NCBI build 36.1), mouse (mm8 NCBI build 36), chicken (galGal3 v2.1 draft assembly Genome Sequencing Center at WUSTL), and Drosophila (dm3 BDGP Release 5). We created Vmatch indexes from Arabidopsis whole-chromosome sequence files from The Arabidopsis Information Resource website (Poole 2007). Likewise, we created Vmatch mapping indexes from $S$. pombe chromosome sequences (ftp://ftp.sanger.ac.uk/pub/yeast/pombe/Chromosome_ contigs/). We required small RNAs to map uniquely to the genome of interest without any mismatches. Sno-derived RNAs were identified by intersecting snoRNA annotations with small RNA data sets using a modified version of the UCSC back end $\mathrm{C}++$ tool, bedIntersect (Karolchik et al. 2008). Conservation profiles for sdRNA were computed using phastCons scores from the phastCons17way and phastCons15way MySQL tables for human and Drosophila snoRNA regions of interest, respectively (Siepel et al. 2005).

The human THP-1 small RNA data set was generated on the Roche FLX Genome Sequencer and contains sequence reads up to $90 \mathrm{nt}$ long. Deep sequencing reads from all other species, however, were generated on the Illumina Genome Analyzer, which has an upper limit of $\sim 30 \mathrm{nt}$ and in many libraries only polled small RNAs $<28$ nt. A complete assessment of sdRNAs $\geq 29$ nt was only possible in human THP-1 cells.

We took advantage of long THP-1 deep sequencing reads to assess the relationship between snoRNA and sdRNA expression. We identified snoRNAs with individual deep sequencing reads covering at least $90 \%$ of their length, pooled them for each snoRNA, and then compared total snoRNA abundance with the abundance of smaller ( $<40 \mathrm{nt})$ sno-derived RNAs.

SdRNA size distributions were assessed using custom AWK, Shell, and Perl scripts. The relative position of sdRNAs within the parent snoRNA was accomplished by parsing individual snoRNAs into $10 \%$ blocks (to normalize for differences in total snoRNA length) and then tabulating the abundance of sdRNA $5^{\prime}$ ends in each decile. SnoRNA enrichments and sdRNA size distributions in mouse wild-type, $d g c r 8 \Delta / \Delta$, and dicer $1 \Delta / \Delta$ ES cells and Drosophila wild-type, $D c r-2^{-l-}$, and $l o q s^{-1-}$ ovaries were performed on libraries normalized against the total abundance of tRNA, snRNA, and srpRNA-derived small RNA fragments (Supplemental Tables 3 and 4), as described previously (Babiarz et al. 2008). We found that 
$\mathrm{t} / \mathrm{sn} /$ srpRNA derived reads are $\sim 2.6 \times$ more highly represented in $d g c r 8 \Delta / \Delta$ and dicer $1 \Delta / \Delta$ ES cells compared to wild-type, and $\sim 1.4 \times$ and $\sim 2 \times$ more abundant in $D c r-2^{-1-}$ and loqs ${ }^{-1-}$ ovaries compared to wild-type, respectively. For all other analyses, we normalized the counts in each library to obtain counts per million, or "tags per million mapped sequences"- the total abundance of the sdRNAs associated with each snoRNA was divided by the total abundance of the library tags that mapped to the genome and multiplied by $10^{6}$. SnoRNAs were only considered in enrichment analyses if their sdRNAs had counts $>5$ in all investigated libraries and had at least one library with sdRNA abundance $>10$. To calculate fold enrichment, the normalized counts of each mutant libraries were divided by the normalized counts of the appropriate WT library. Previous analyses have normalized libraries using the number of small RNA “degradation products," including sequences derived from snoRNAs. We reasoned that a direct comparison of the relative sequence depths of the libraries would be a more accurate measure of abundance, particularly in light of the fact that it appears that small RNAs can be derived from longer small RNA species including snoRNAs and tRNAs.

Orthologous sdRNAs were identified by mapping human and chicken sdRNAs to the mouse genome using the UCSC syntenic alignment tool, liftOver, and intersecting them with previously mapped mouse sdRNAs. For sdRNAs to be considered orthologous, we required that the $5^{\prime}$ ends of the sdRNAs from each species map to the same position in the mouse snoRNA, a $>90 \%$ sequence identity, and similarity in size $( \pm 20 \%)$.

SdRNA 5 ' nucleotide enrichments were assessed by extracting the sequences of interest, parsing the sequences by size, and tabulating the percentage of each base using the Biopieces program weight_matrix. RNA structures (see Fig. 3) were calculated using the Vienna RNA fold web server (http://rna.tbi.univie.ac.at/cgi-bin/ RNAfold.cgi) (Hofacker 2003).

\section{SUPPLEMENTAL MATERIAL}

Supplemental material can be found at http://www.rnajournal.org.

\section{ACKNOWLEDGMENTS}

We thank Martin Hansen for access to, and assistance with, the Biopieces toolset. R.J.T. is supported by a National Science Foundation Graduate Research Fellowship. J.S.M. is supported by an Australian Research Council Federation Fellowship (FF0561986), the University of Queensland, and the Queensland State Government. This work was also supported by grants for the Genome Network Project from the Ministry of Education, Culture, Sports, Science and Technology, Japan (Y.H.), and a Research Grant for the RIKEN Genome Exploration Research Project from the Ministry of Education, Culture, Sports, Science and Technology of the Japanese Government, the RIKEN Frontier Research System, Functional RNA Research Program (Y.H.).

Received December 22, 2008; accepted April 9, 2009.

\section{REFERENCES}

Babiarz JE, Ruby JG, Wang Y, Bartel DP, Blelloch R. 2008. Mouse ES cells express endogenous shRNAs, siRNAs, and other Micropro- cessor-independent, Dicer-dependent small RNAs. Genes \& Dev 22: 2773-2785.

Brown JW, Clark GP, Leader DJ, Simpson CG, Lowe T. 2001. Multiple snoRNA gene clusters from Arabidopsis. RNA 7: 1817-1832.

Buhler M, Spies N, Bartel DP, Moazed D. 2008. TRAMP-mediated RNA surveillance prevents spurious entry of RNAs into the Schizosaccharomyces pombe siRNA pathway. Nat Struct Mol Biol 15: 1015-1023.

Czech B, Malone CD, Zhou R, Stark A, Schlingeheyde C, Dus M, Perrimon N, Kellis M, Wohlschlegel JA, Sachidanandam R, et al. 2008. An endogenous small interfering RNA pathway in Drosophila. Nature 453: 798-802.

Drysdale R. 2008. FlyBase: A database for the Drosophila research community. Methods Mol Biol 420: 45-59.

Ender C, Krek A, Friedlander MR, Beitzinger M, Weinmann L, Chen W, Pfeffer S, Rajewsky N, Meister G. 2008. A human snoRNA with microRNA-like functions. Mol Cell 32: 519-528.

Flicek P, Aken BL, Beal K, Ballester B, Caccamo M, Chen Y, Clarke L, Coates G, Cunningham F, Cutts T, et al. 2008. Ensembl 2008. Nucleic Acids Res 36: D707-D714.

Gaspin C, Cavaille J, Erauso G, Bachellerie JP. 2000. Archaeal homologs of eukaryotic methylation guide small nucleolar RNAs: Lessons from the Pyrococcus genomes. J Mol Biol 297: 895-906.

Ghildiyal M, Seitz H, Horwich MD, Li C, Du T, Lee S, Xu J, Kittler EL, Zapp ML, Weng Z, et al. 2008. Endogenous siRNAs derived from transposons and mRNAs in Drosophila somatic cells. Science 320: 1077-1081.

Glazov EA, Cottee PA, Barris WC, Moore RJ, Dalrymple BP, Tizard ML. 2008. A microRNA catalog of the developing chicken embryo identified by a deep sequencing approach. Genome Res 18: 957-964.

Hertz-Fowler C, Peacock CS, Wood V, Aslett M, Kerhornou A, Mooney P, Tivey A, Berriman M, Hall N, Rutherford K, et al. 2004. GeneDB: A resource for prokaryotic and eukaryotic organisms. Nucleic Acids Res 32: D339-D343.

Hock J, Weinmann L, Ender C, Rudel S, Kremmer E, Raabe M, Urlaub H, Meister G. 2007. Proteomic and functional analysis of Argonaute-containing mRNA-protein complexes in human cells. EMBO Rep 8: 1052-1060.

Hofacker IL. 2003. Vienna RNA secondary structure server. Nucleic Acids Res 31: 3429-3431.

Hutvagner G, Simard MJ. 2008. Argonaute proteins: Key players in RNA silencing. Nat Rev Mol Cell Biol 9: 22-32.

Karolchik D, Kuhn RM, Baertsch R, Barber GP, Clawson H, Diekhans M, Giardine B, Harte RA, Hinrichs AS, Hsu F, et al. 2008. The UCSC Genome Browser Database: 2008 update. Nucleic Acids Res 36: D773-D779.

Kiss T. 2002. Small nucleolar RNAs: An abundant group of noncoding RNAs with diverse cellular functions. Cell 109: 145-148.

Li CF, Pontes O, El-Shami M, Henderson IR, Bernatavichute YV, Chan SW, Lagrange T, Pikaard CS, Jacobsen SE. 2006. An ARGONAUTE4-containing nuclear processing center colocalized with Cajal bodies in Arabidopsis thaliana. Cell 126: 93-106.

Li CF, Henderson IR, Song L, Fedoroff N, Lagrange T, Jacobsen SE. 2008. Dynamic regulation of ARGONAUTE4 within multiple nuclear bodies in Arabidopsis thaliana. PLoS Genet 4: e27. doi: 10.1371/journal.pgen.0040027.

Matera AG, Terns RM, Terns MP. 2007. Noncoding RNAs: Lessons from the small nuclear and small nucleolar RNAs. Nat Rev Mol Cell Biol 8: 209-220.

Montgomery TA, Howell MD, Cuperus JT, Li D, Hansen JE, Alexander AL, Chapman EJ, Fahlgren N, Allen E, Carrington JC. 2008. Specificity of ARGONAUTE7-miR390 interaction and dual functionality in TAS3 trans-acting siRNA formation. Cell 133: $128-141$.

Morin RD, O'Connor MD, Griffith M, Kuchenbauer F, Delaney A, Prabhu AL, Zhao Y, McDonald $\mathrm{H}$, Zeng T, Hirst $\mathrm{M}$, et al. 2008. Application of massively parallel sequencing to microRNA 


\section{Taft et al.}

profiling and discovery in human embryonic stem cells. Genome Res 18: 610-621.

Omer AD, Lowe TM, Russell AG, Ebhardt H, Eddy SR, Dennis PP. 2000. Homologs of small nucleolar RNAs in Archaea. Science 288: $517-522$.

Peters L, Meister G. 2007. Argonaute proteins: Mediators of RNA silencing. Mol Cell 26: 611-623.

Pontes O, Pikaard CS. 2008. siRNA and miRNA processing: new functions for Cajal bodies. Curr Opin Genet Dev 18: 197-203.

Pontes O, Li CF, Nunes PC, Haag J, Ream T, Vitins A, Jacobsen SE, Pikaard CS. 2006. The Arabidopsis chromatin-modifying nuclear siRNA pathway involves a nucleolar RNA processing center. Cell 126: 79-92.

Poole RL. 2007. The TAIR database. Methods Mol Biol 406: 179-212.

Ruby JG, Stark A, Johnston WK, Kellis M, Bartel DP, Lai EC. 2007. Evolution, biogenesis, expression, and target predictions of a substantially expanded set of Drosophila microRNAs. Genome Res 17: 1850-1864.

Saraiya AA, Wang CC. 2008. snoRNA, a novel precursor of microRNA in Giardia lamblia. PLoS Pathog 4: e1000224. doi: 10.1371/journal. ppat.1000224.
Siepel A, Bejerano G, Pedersen JS, Hinrichs AS, Hou M, Rosenbloom K, Clawson H, Spieth J, Hillier LW, Richards S, et al. 2005. Evolutionarily conserved elements in vertebrate, insect, worm, and yeast genomes. Genome Res 15: 1034-1050.

Taft RJ, Glazov EA, Cloonan N, Simons C, Stephen S, Faulkner GJ, Lassmann T, Forrest ARR, Grimmond SM, Schroder K, et al. 2009. Tiny RNAs associated with transcription start sites in animals. Nat Genet 41: 572-578.

Watanabe T, Totoki Y, Toyoda A, Kaneda M, KuramochiMiyagawa S, Obata Y, Chiba H, Kohara Y, Kono T, Nakano T, et al. 2008. Endogenous siRNAs from naturally formed dsRNAs regulate transcripts in mouse oocytes. Nature 453: 539543.

Wheeler DL, Barrett T, Benson DA, Bryant SH, Canese K, Chetvernin V, Church DM, Dicuccio M, Edgar R, Federhen S, et al. 2008. Database resources of the National Center for Biotechnology Information. Nucleic Acids Res 36: D13-D21.

Wu H, Neilson JR, Kumar P, Manocha M, Shankar P, Sharp PA, Manjunath N. 2007. miRNA profiling of naive, effector and memory CD8 T cells. PLoS One 2: e1020. doi: 10.1371/journal.pone. 0001020 

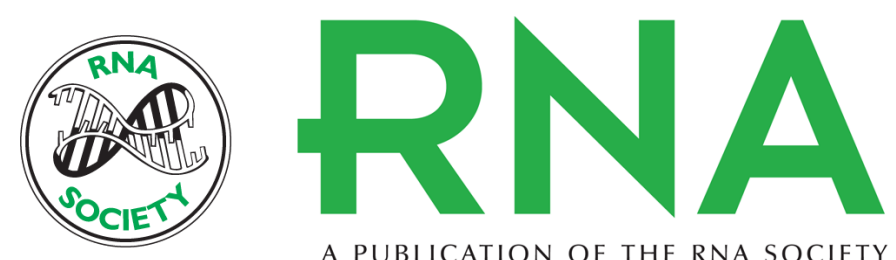

A PUBLICATION OF THE RNA SOCIETY

\section{Small RNAs derived from snoRNAs}

Ryan J. Taft, Evgeny A. Glazov, Timo Lassmann, et al.

RNA 2009 15: 1233-1240 originally published online May 27, 2009

Access the most recent version at doi:10.1261/rna.1528909

Supplemental
Material http://rnajournal.cshlp.org/content/suppl/2009/05/28/rna.1528909.DC1

References This article cites 33 articles, 8 of which can be accessed free at:

http://rnajournal.cshlp.org/content/15/7/1233.full.html\#ref-list-1

License

Email Alerting Receive free email alerts when new articles cite this article - sign up in the box at the Service top right corner of the article or click here.

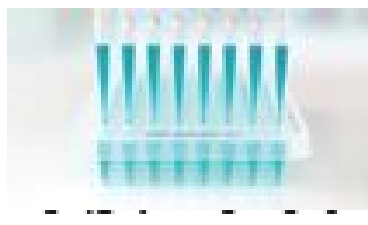

\section{Providing Precise Solutions for} your research.

To subscribe to RNA go to:

http://rnajournal.cshlp.org/subscriptions 